\title{
Die Patientenverfügung - Ausdruck gesundheitlicher Selbstbestimmung - und ihre Bindungswirkung für Ärzte
}

\author{
The Living Will Declaration - Expression of Self-Determination in Health-Care - \\ and its Legal Validity for Physicians
}

Autor

W. G. Leisner

eingereicht 27.6.2010

akzeptiert nach Revision

1. 7.2010

Bibliografie

DOI http://dx.doi.org/

10.1055/s-0030-1255626

Pneumologie 2010; 64:

712-713 @ Georg Thieme

Verlag KG Stuttgart · New York

ISSN 0934-8387

Korrespondenzadresse

Priv.-Doz. Dr.

Walter Georg Leisner

Universität Hamburg

Rechtsanwalt

Leisner, Zehentmeier \& Kollegen

Rechtsanwälte Steuerberater

Widenmayerstr 9

80538 München

leisner@|z-legal.de

\section{Zusammenfassung \\ $\nabla$}

Die Patientenverfügung ist seit 1.9.2009 in $\S \S 1901$ a - c BGB gesetzlich geregelt. Sie ist ohne Wahrung bestimmter gesetzlicher Formvorschriften schriftlich zu verfassen. Hiervon abzugrenzen ist der Behandlungswunsch, nach dem der Betreuer zu entscheiden hat, ob er in eine ärztliche Maßnahme einwilligt oder sie untersagt. Die Patientenverfügung bindet den Arzt für konkrete behandlungsbezogene Wünsche und gilt unabhängig von Art und Stadium der Erkrankung des Patienten, da das Grundrecht auf Selbstbestimmung (Art. 2 Abs. 2 GG) auch das Recht zu sterben beinhaltet. Da der Arzt verpflichtet ist, den Patientenwillen zu beachten, muss er bei Nichtbefolgung der Patientenverfügung mit haftungsund strafrechtlichen Folgen rechnen.

\section{Gegenstand und Form der Patientenverfügung \\ $\nabla$}

Das bisher lediglich richterrechtlich fortgebildete Recht der Patientenverfügung ist nun seit 1. 9. 2009 in $\S \S 1901 \mathrm{a}$ - c des Bürgerlichen Gesetzbuchs gesetzlich festgelegt. Nach $\S 1901$ a Abs. 1 Satz 1 BGB ist eine Patientenverfügung die schriftliche Festlegung eines einwilligungsfähigen Volljährigen für den Fall seiner Einwilligungsunfähigkeit, ob er in bestimmte, zum Zeitpunkt der Festlegung noch nicht unmittelbar bevorstehende Untersuchungen seines Gesundheitszustands, Heilbehandlungen oder ärztliche Eingriffe einwilligt oder sie untersagt. Der Patient übt damit sein Selbstbestimmungsrecht im Hinblick auf seine künftige Behandlung aus. Eine notarielle Beurkundung ist hierbei nicht notwendig; auch ist die Angabe von Zeit und Ort für die Wirksamkeit der Patientenverfügung nicht erforderlich. Der Widerruf der Patientenverfügung ist jederzeit formlos möglich, § 1901 a Abs. 1 Satz 4 BGB.

\section{Abstract \\ $\nabla$}

Since September $1^{\text {st }} 2009$, the Living Will Declaration is regulated by law as held in $\S \S 1901 \mathrm{a}-\mathrm{c}$ BGB. It does not need a certain format except for being in the written form. This must be distinguished from the right to choose a certain treatment according to the medical attendant and his intention to agree to or forbid a certain treatment. The Living Will Declaration binds the physician to certain clearly specified procedures that the patient has agreed to and is valid independently from the nature of the patient's illness or its stage because the basic right for self-determination (Art. 2 Abs. 2 GG) includes the right to die. As the physician is committed to observe the patient's will, he will have to face legal consequences in terms of liability and criminal law if he disregards the Living Will Declaration.

\section{Bindungswirkung der Patienten- verfügung \\ $\nabla$}

\section{Behandlungswunsch}

Abzugrenzen von der Patientenverfügung ist der Behandlungswunsch gemäß §1901 a Abs. 2 Satz 1 $B G B$, nach dem der (ärztliche) Betreuer die Behandlungswünsche oder den mutmaßlichen Willen des Betreuten festzustellen und auf dieser Grundlage zu entscheiden hat, ob er in eine ärztliche Maßnahme nach Abs. 1 einwilligt oder sie untersagt, wenn keine Patientenverfügung vorliegt oder die Festlegungen einer Patientenverfügung nicht auf die aktuelle Lebens- und Behandlungssituation zutreffen.

\section{Bindung des Arztes}

Die Bindungswirkung einer Patientenverfügung tritt dann ein, wenn zwischen der in der Patientenverfügung enthaltenen Einwilligung bzw. Untersagung derselben und dem ärztlichen Eingriff eine gewisse Übereinstimmung besteht. Dabei 
bindet die Patientenverfügung nur den Arzt, nicht aber den Patienten, da sie nur solange gilt, bis er seine Erklärung ändert oder konkrete Anhaltspunkte für eine Willensänderung vorliegen. Für den Arzt ergibt sich die Verbindlichkeit einer antizipierten Einwilligung für konkrete behandlungsbezogene Wünsche aus den allgemeinen Grundsätzen des Medizinrechts für die Einwilligung in ärztliche Maßnahmen [1].

\section{Auslegung}

Dabei bedarf jede Patientenverfügung der Auslegung. Da sich der Patient zu der Behandlungsmaßnahme nicht mehr äußern kann, ist die frühere Erklärung heranzuziehen und auszulegen, was der Patient mit seiner Patientenverfügung für die in der aktuellen Behandlungssituation anstehende Entscheidung über die ärztliche Maßnahme erklären wollte. Hierzu gehört auch die Pflicht, nahestehende Personen und Angehörige des Patienten einzubeziehen. Bei einer Entscheidung auf Grundlage des mutmaßlichen Willens ist allein auf individuelle konkrete Kriterien zurückzugreifen, damit Entscheidungen am Lebensende wirklich die erforderliche Rückbindung an das Selbstbestimmungsrecht der Betroffenen wahren [2].

\section{Reichweite}

Gemäß § 1901 a Abs. 3 BGB gilt die Patientenverfügung unabhängig von Art und Stadium der Erkrankung des Patienten. Denn bei einer Reichweitenbegrenzung wäre es äußerst zweifelhaft, ob einem Arzt eine sichere Entscheidung darüber möglich sei, ob der Patientenwille beachtet werden darf oder ob der Patient den Eingriff gegen seinen Willen erdulden muss.

Nach der gesetzlichen Regelung wird darauf vertraut, dass der mündige Bürger eine Verfügung nur mit dem Inhalt errichtet, den er auch tatsächlich vor sich verantworten kann, und dass er sich Rat einholen wird, dem er für eine verantwortungsbewusste Entscheidung über die Art seines Lebensendes bedarf.

Als Kompensation für den Wegfall der Reichweitenbegrenzung und den Verzicht auf eine verbindliche Beratung wurde gemäß $\S 1901$ b BGB ein Gespräch zur Feststellung des Patientenwillens festgelegt. Hier muss der Arzt in einem Gespräch mit dem Vertreter des Patienten zu einer Entscheidung gelangen, die dem mutmaßlichen Patientenwillen entspricht. Dabei soll eine Abwägung zwischen Lebensschutz und Selbstbestimmungsrecht des Patienten stattfinden.

Ohne die Einwilligung des Betreuers bzw. Bevollmächtigten darf der Arzt keine medizinisch indizierte Maßnahme unterlassen, auch wenn eine Patientenverfügung vorliegt, die einen entsprechenden Willen des Bevollmächtigten nahelegt. Denn die Patientenverfügung kann ärztliches Handeln nicht rechtfertigen [3]. Nicht der Arzt, sondern der Betreuer bzw. Bevollmächtigte entscheidet, ob und welche der getroffenen Festlegungen auf die konkrete Lebens- und Behandlungssituation zutreffen, $\S 1901$ a-c BGB.

Auch hier gelten die allgemeinen Grenzen der Patientenautonomie ( $\S \S 134,138$ BGB), sodass niemand einen anderen mittels einer Patientenverfügung von dem strafrechtlichen Verbot der
Tötung auf Verlangen ( $\$ 216$ StGB) freistellen oder einen Anspruch auf eine nicht indizierte oder gar kontra-indizierte ärztliche Maßnahme begründen kann [4].

Das Grundrecht auf (gesundheitliche) Selbstbestimmung, Art. 2 Abs. 2 GG, beinhaltet auch das Recht zu sterben, sodass ein Behandlungsveto eines Patienten vom Arzt beachtet werden muss, auch wenn die Nichtbehandlung zum Tode führt [2].

\section{Strafbarkeit/Haftung bei Nichteinhaltung der Patientenverfügung}

Bei Nichtbeachtung der Patientenverfügung muss der Arzt mit haftungs- und strafrechtlichen Folgen rechnen, denn der Arzt ist verpflichtet, den Patientenwillen zu beachten.

So kann der Arzt wegen Körperverletzung, bei unterlassener Behandlung oder nicht gerechtfertigtem Abbruch lebenserhaltender Maßnahmen wegen eines Tötungsdeliktes oder unterlassener Hilfeleistung strafrechtlich verfolgt werden [5].

Eine Strafbarkeit wegen fahrlässiger Körperverletzung gemäß §229 StGB liegt dabei nicht vor, da hier keine Sorgfaltspflichtverletzung darin gesehen werden kann, dass der Arzt eine Behandlung vornimmt, ohne zuvor Nachforschungen über das Vorhandensein einer Patientenverfügung angestellt zu haben, da diesen insoweit keine Nachforschungspflicht trifft [6].

Ebenso kommt eine Haftung gemäß § 823 BGB in Betracht, die zu zivilrechtlichen Schadenersatz- und Schmerzensgeldansprüchen führt.

\section{Interessenkonflikt \\ $\nabla$}

Durch die Festschreibung der Patientenverfügung ins Gesetz ergibt sich nun ein grundsätzlicher Konflikt zwischen dem Selbstbestimmungsrecht und dem Integritätsschutz des Patienten, da ein Behandlungsveto eines Patienten beachtet werden muss, auch wenn dies zum Tode führt. Neben dem Abwehrrecht ist jedoch auch die Dimension eines staatlichen Schutzrechts aus Art. 2 Abs. 2 GG zu beachten. Dies ist auch Gegenstand der Kritik der gesetzlichen Festschreibung der Patientenverfügung. Aufgrund dieser Kollision sollte zumindest eine fachkundige (notarielle oder anwaltschaftliche) Beratung der Patientenverfügung für ihre Wirksamkeit vorausgehen [2].

\section{Literatur}

1 Laufs A, Katzenmeier C, Lipp V. Arztrecht. C. H. Beck, 2009: Rn. 150

2 Höfling $W$. Das neue Patientenverfügungsgesetz. Neue Juristische Wochenschrift (NJW) 2009: 2849-2852

3 Diehn T, Rebhan R. Vorsorgevollmacht und Patientenverfügung. NJW 2010: $326-331$

4 Laufs A, Katzenmeier C, Lipp V. Arztrecht. C. H. Beck, 2009: Rn. 134

5 BVerfG. Beschl. vom 30.1.2002-2 BvR 1451/01, NStZ-RR 2002: S. $169 \mathrm{ff}$

6 Hartmann T. Patientenverfügung und Psychiatrische Verfügung - Verbindlichkeit für den Arzt? Neue Strafrecht Zeitung (NStZ) 2000: 113120 